EPJ Web of Conferences 49, 15014 (2013)

DOI: $10.1051 /$ epjconf/20134915014

(C) Owned by the authors, published by EDP Sciences, 2013

\title{
Search for SUSY in events with R-parity violation or long-lived particles at ATLAS
}

\author{
Shingo Kazama ${ }^{1, a}$ On behalf of the ATLAS Collaboration \\ ${ }^{1}$ The University of Tokyo, Japan
}

\begin{abstract}
This paper presents the latest results of searches for supersymmetry in events with R-parity violation or long-lived particles with the ATLAS detector. The data were recorded in $\sqrt{s}=7(8) \mathrm{TeV}$ proton-proton collisions at the LHC in 2011(2012) and correspond to a total integrated luminosity of 5(13) $\mathrm{fb}^{-1}$, respectively. No excess above the Standard Model background expectation is observed, thus stringent limits are set on the various SUSY models.
\end{abstract}

\section{Introduction}

Supersymmetry (SUSY) [1-9] is an attractive theory for physics beyond the Standard Model (SM). R-parity is a quantum number defined as $(-1)^{3(B-L)+2 S}$, where $\mathrm{B}, \mathrm{L}$ and $\mathrm{S}$ are the baryon number, lepton number, and spin quantum numbers, respectively. R-parity is frequently assumed to be a conserved quantity, which leaves the lightest supersymmetric particle(LSP) stable. However, SUSY does not necessarily require R-parity conservation. If the Rparity violating (RPV) couplings are allowed, such models may lead to distinctive experimental signatures which are not present in SM. Meta-stable or long-lived particles also provide unique detector signatures which are free of SM backgrounds. In SUSY, non-prompt particle decay can be caused by i) very weak R-Parity violation, i.e. one of the Yukawa coupling $\lambda_{\mathrm{ijk}}, \lambda_{\mathrm{ijk}}^{\prime}$ or $\lambda_{\mathrm{ijk}}^{\prime \prime} \sim \mathrm{O}\left(10^{-5}\right)$, where the indices $i, j$ and $k$ refer to quark and lepton generations, ii) low mass difference between chargino and neutralino $\left(\Delta m_{\tilde{\chi}_{1}^{0}}\right)$ or iii) very weak coupling to the gravitino in GMSB models [10,11]. All these possibilities have been explored with the ATLAS detector [12]. This paper summarizes the results of these searches based on $5 \mathrm{fb}^{-1}$ of $\sqrt{s}=7 \mathrm{TeV}$ data or $13 \mathrm{fb}^{-1}$ of $\sqrt{s}=8 \mathrm{TeV}$ data.

\section{Search for SUSY in events with four or more leptons}

If one of the $\lambda_{\mathrm{ijk}}$ is non-zero, final states with high lepton multiplicities can be produced due to the decay of neutralino LSP into two charged leptons and one neutrino. In this analysis, only one RPV coupling, either $\lambda_{121}$ or $\lambda_{122}$, is set to be non-zero. First of all, a signal region (SR) is defined with the requirement that events contain four or more leptons (electrons, muons). After this requirement, the invariant mass of any same-flavour opposite-sign

\footnotetext{
ae-mail: skazama@cern.ch
}

(SFOS) lepton pair is required to be greater than $12 \mathrm{GeV}$ to suppress the low-mass Drell-Yan backgrounds. Z-boson candidates are also vetoed by removing events with pairs, triplets or quadruplets of leptons with an invariant mass inside the $10 \mathrm{GeV}$ interval around the $\mathrm{Z}$ mass pole. Lepton pairs and triplets considered for this Z-veto must contain a SFOS pair, while lepton quadruplets must consist of two SFOS pairs. As a final selection, $E_{\mathrm{T}}^{\text {miss }}>50 \mathrm{GeV}$ is required to the candidate events. No significant excess of events is found in the signal region as shown in Fig. 1, so limits are placed on various R-parity violating simplified models. In the Wino simplified model of RPV SUSY [13], chargino masses up to $710 \mathrm{GeV}$ are excluded (Fig. 2) [14].

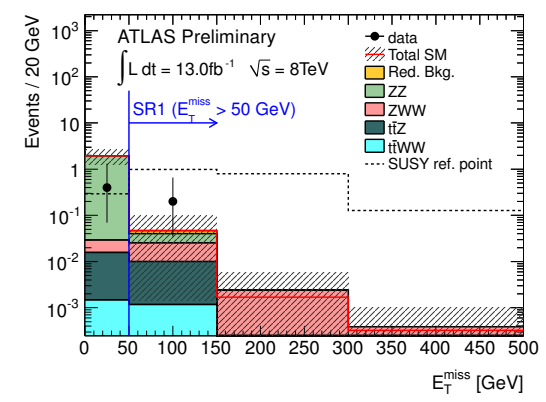

Figure 1. Distribution of $E_{\mathrm{T}}^{\text {miss }}$ in events with at least four leptons and no $\mathrm{Z}$ boson candidates [14]. The uncertainty band includes both statistical and systematic uncertainties. SUSY ref. point is a scenario from the RPV Wino $\lambda_{121}$ simplified model, with $\mathrm{m}_{\tilde{\chi}_{1}^{ \pm}}$ $=600 \mathrm{GeV}, \mathrm{m}_{\tilde{\chi}_{1}^{0}}=400 \mathrm{GeV}$.

\section{Search for SUSY in events with a heavy narrow resonance decaying to $e-\mu$}

R-parity violating SUSY models allow couplings which violate lepton flavor conservation. Here, we present the re- 


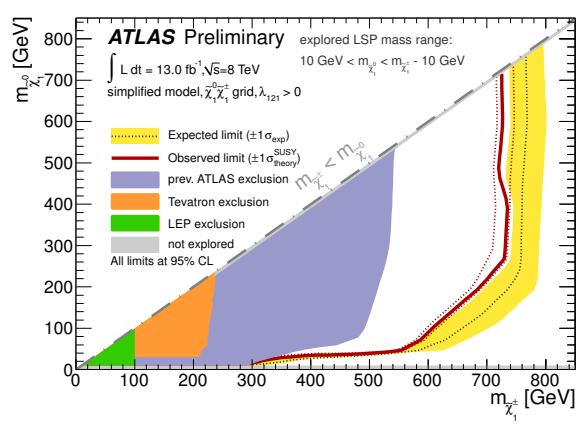

Figure 2. Observed and expected 95\% CL limit contours for the RPV Wino simplified model where $\lambda_{121}>0$ [14].

sults of a search for a new heavy neutral particle decaying to an $e^{ \pm} \mu^{\mp}$ pair [15]. In RPV SUSY, this signature could arise via the $\tau$ sneutrino production and decay via the RPV coupling $\lambda_{132}$ and $\lambda_{311}^{\prime}$, respectively. Candidate events are selected by scanning the invariant mass of the $e^{ \pm} \mu^{\mp}$ pair depending on the $\tau$ sneutrino mass (Fig. 3). No significant excess above the SM expectation is observed, and exclusions at 95\% CL are placed on the cross section times branching ratio for the production of an R-parity-violating supersymmetric $\tau$ sneutrino (Fig. 4). For couplings $\lambda_{311}^{\prime}=$ 0.10 and $\lambda_{132}=0.05$, the $\tau$ sneutrino is excluded at $95 \%$ CL for a mass up to $1610 \mathrm{GeV}$.

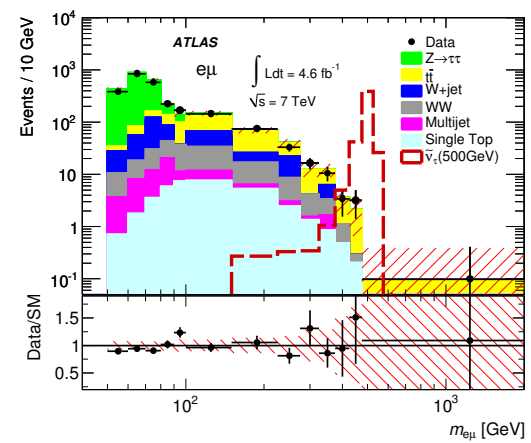

Figure 3. Observed and predicted invariant mass of $e^{ \pm} \mu^{\mp}$ pair [15]. Signal simulation is shown for $m_{\tilde{v}_{\tau}}=500 \mathrm{GeV}\left(\lambda_{311}^{\prime}=\right.$ $\left.0.11, \lambda_{132}=0.07\right)$.

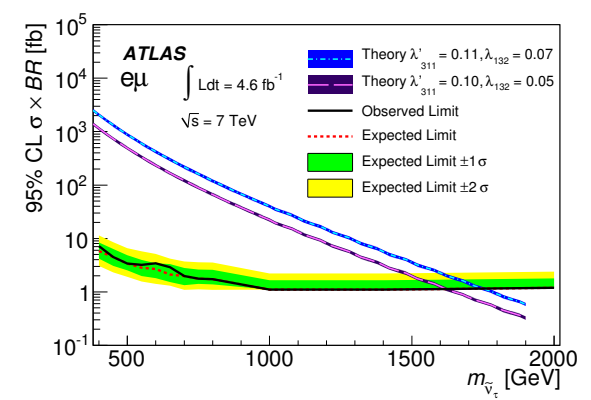

Figure 4. The 95\% CL upper limit on the production cross section times branching ratio as a function of $\tau$ sneutrino mass [15].

\section{Search for pair production of massive particles decaying into three quarks}

A search for fully hadronic final states involving massive particle decays to three jets is performed [16]. An RPV SUSY model in which pair produced gluinos each decay to three jets via an off-shell squark $\left(\tilde{g} \rightarrow q \tilde{q} \rightarrow\right.$ qqq with $\mathrm{m}_{\tilde{q}}$ $\left.\gg \mathrm{m}_{\tilde{\mathrm{g}}}\right)$ is used as a benchmark physics model. The analysis is divided into two search channels, each optimized separately for their sensitivity to high-mass and low-mass gluino production. The first search channel uses a stringent selection on the transverse momentum of the six leading jets and is performed as a counting experiment. The second search channel focuses on low-mass gluinos produced with a large boost. Large-radius jets are selected and the invariant mass of each of the two leading jets is used as a discriminant between the signal and the background. The results are found to be consistent with SM expectations and limits are set on the allowed gluino mass. For the resolved analysis channel, gluino with masses up to $666 \mathrm{GeV}$ are excluded at 95\% CL (Fig. 5). For the boosted analysis channel, gluino with masses up to 255 $\mathrm{GeV}$ are excluded at 95\% CL (Fig. 6).

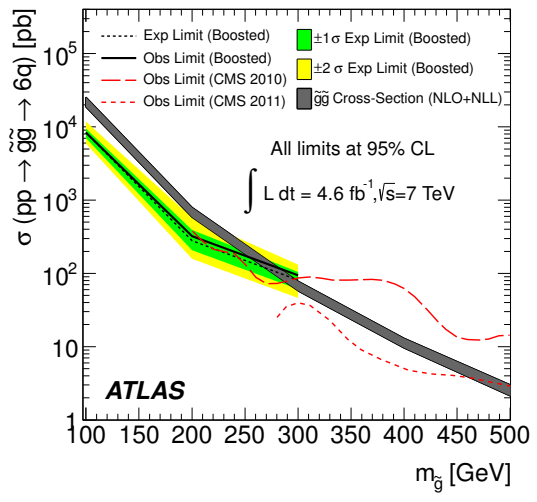

Figure 5. The expected and observed 95\% CL limits for the resolved analyses channel [16].

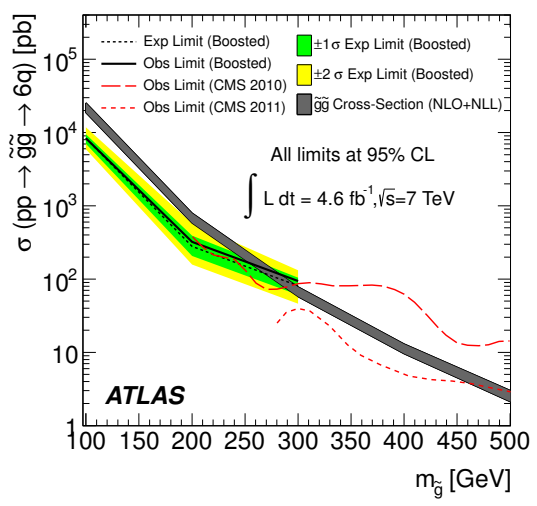

Figure 6. The expected and observed 95\% CL limits for the boosted analyses channel [16]. 


\section{Search for long-lived, heavy particles in final states with a muon and multi-track displaced vertex}

An analysis searching for displaced vertices (DVs) with several tracks and one muon is performed [17]. Such a signature could arise from a neutralino LSP decaying as $\tilde{\chi}_{1}^{0} \rightarrow \tilde{\mu} \mu \rightarrow \mu q_{\mathrm{i}} q_{\mathrm{j}}$ via an intermediate virtual $\tilde{\mu}$ and the RPV coupling $\lambda_{2 \mathrm{ij}}^{\prime}$. A background-free signal region is chosen in the $\mathrm{M}_{\mathrm{DV}}-\mathrm{N}_{\text {tracks }}$ plane, where $\mathrm{M}_{\mathrm{DV}}\left(\mathrm{N}_{\text {tracks }}\right)$ is the reconstructed mass (number of tracks) of the displaced vertex (Fig. 7). To increase the sensitivity to SUSY signals, a dedicated track reconstruction for particles from the displaced vertex has been developed. This analysis, rather model independent, is sensitive to heavy particles that decay at radial distances between 4 and $180 \mathrm{~mm}$ from the pp interaction point in association with a high $p_{\mathrm{T}}$ muon. No events in the signal region are observed, so limits are set on the product of di-squark production cross section and decay chain branching fraction squared, in a SUGRA scenario where the lightest neutralino produced in the primary-squark decay undergoes an R-parity violating decay into a muon and two quarks (Fig. 8).

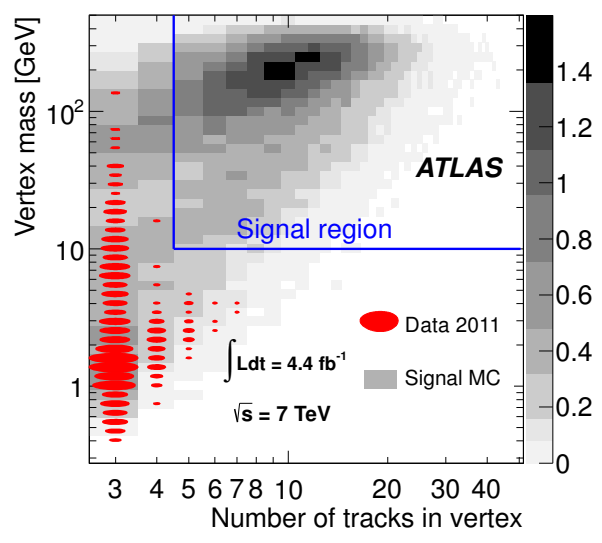

Figure 7. Vertex mass $\left(\mathrm{m}_{\mathrm{DV}}\right)$ vs vertex track multiplicity $\left(\mathrm{N}_{\text {tracks }}\right.$ ) for DVs for candidate events and signal samples [17].

\section{Search for direct chargino production in anomaly-mediated SUSY breaking models}

A search for direct chargino production in anomalymediated SUSY breaking (AMSB) scenarios[18, 19] is performed [20]. In this model, the lightest chargino is predicted to have a lifetime long enough to be detected in the tracking detectors of collider experiments. This analysis explores such models by searching for chargino decays that result in tracks with few associated hits in the outer region of the tracking system, which is covered by the transition radiation tracker (TRT). Fig. 8 shows the distribution of the number of hits along the track trajectory in the outer layers of the TRT $\left(N_{\mathrm{TRT}}^{\text {outer }}\right)$ for data and signal events. If charginos decay before reaching the TRT outer

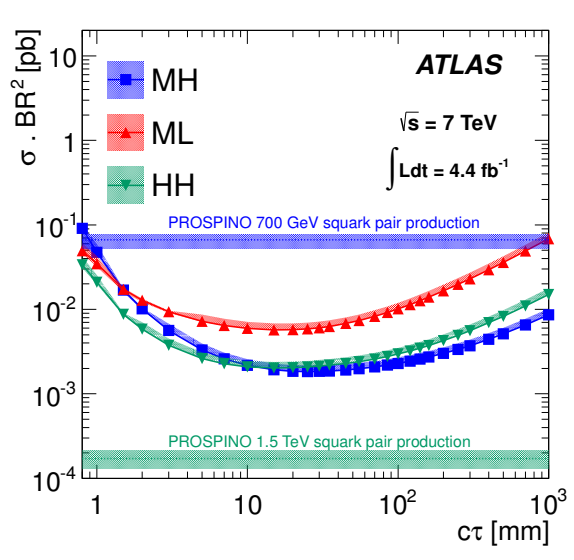

Figure 8. Upper limits at $95 \%$ confidence level on $\sigma \cdot B R^{2}$ vs the neutralino lifetime for different combinations of squark and neutralino masses [17]. The shaded areas around these curves represent the $\pm 1 \sigma$ uncertainty bands on the expected limits.

module, $N_{\mathrm{TRT}}^{\text {outer }}$ is expected to have a value near zero; conversely, SM charged particles traversing the TRT typically have $N_{\text {TRT }}^{\text {outer }} \simeq 15$. In order to enhance the expected signal to background ratio, $N_{\mathrm{TRT}}^{\text {outer }}<5$ is required.

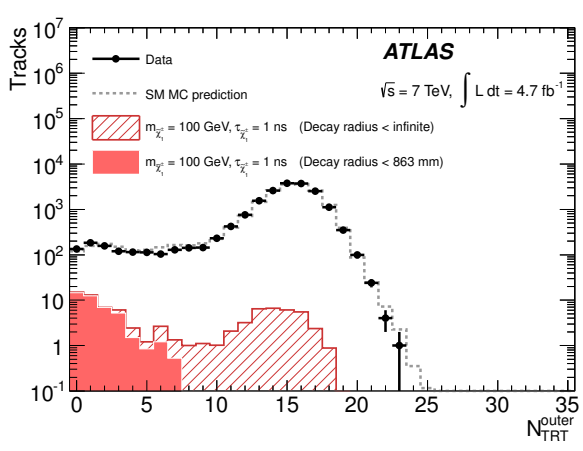

Figure 9. Distribution of the number of hits in the outer layers of the TRT for data and signal events $\left(\mathrm{m}_{\tilde{\chi}_{1}^{ \pm}}=100 \mathrm{GeV}, \tau_{\tilde{\chi}_{1}^{ \pm}}=1 \mathrm{~ns}\right)$ [20].

According to MC simulation, there are two types of background in this analysis. One is high- $p_{\mathrm{T}}$ charged hadrons which interact with the material in the TRT detector. The other is electrons failing to satisfy their identification criteria due to large bremsstrahlung. A background estimation based on the MC simulation suffers from large uncertainties due to low numbers of tracks after all the selection requirements and has difficulty in simulating the properties of these background tracks. Therefore, an approach using data-driven control samples enriched in these background categories is employed to estimate the background track $p_{\mathrm{T}}$ spectrum. A simultaneous fit is then performed for signal and background yields using the $p_{\mathrm{T}}$ spectrum of observed tracks.

Fig. 10 shows the $p_{\mathrm{T}}$ distribution for the selected data events compared to the background model obtained by the "background-only" fit in the $p_{\mathrm{T}}$ range above $10 \mathrm{GeV}$. The 
results are found to be consistent with SM expectations and limits are set on the minimal AMSB model. Fig. 11 shows the constraint on the $\Delta m_{\tilde{\chi}_{1}^{0}}-m_{\tilde{\chi}_{1}^{ \pm}}$parameter space of the minimal AMSB model. For $\Delta m_{\tilde{\chi}_{1}^{0}}=160 \mathrm{MeV}$, the value most probable in the model, a new limit of $m_{\tilde{\chi}_{1}^{ \pm}}>$ $103 \mathrm{GeV}$ at $95 \% \mathrm{CL}$ is obtained. For $\Delta m_{\tilde{\chi}_{1}^{0}} \sim 140 \mathrm{MeV}$, a more stringent limit of $m_{\tilde{\chi}_{1}^{ \pm}}>260 \mathrm{GeV}$ is set.

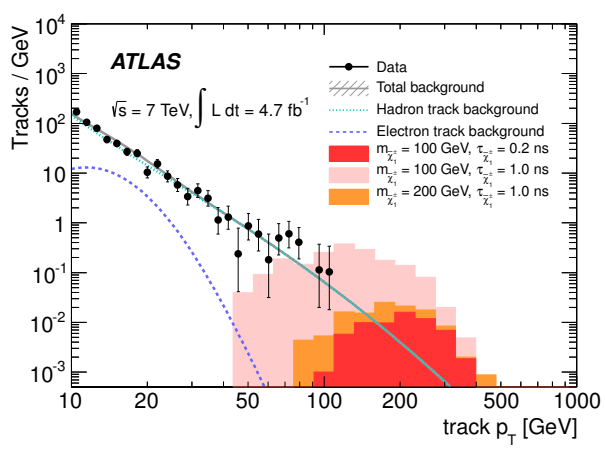

Figure 10. The $p_{\mathrm{T}}$ distribution of candidate tracks [20]. The solid circles show data and lines show background shapes obtained using the "background-only" fit.

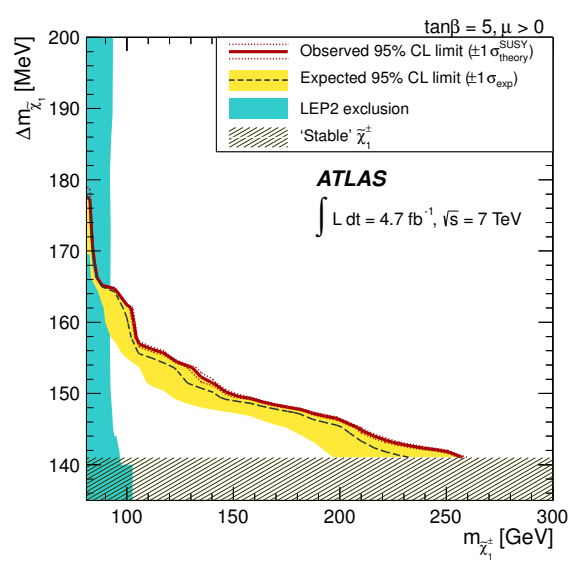

Figure 11. The constraint on the $\Delta m_{\tilde{\chi}_{1}^{0}}-m_{\tilde{\chi}_{1}^{ \pm}}$space for $\tan \beta=5$ and $\mu>0$ [20].

\section{Searches for heavy long-lived sleptons and R-hadrons}

An analysis searching for stable massive particles (SMPs) is performed [21]. SMPs, such as long-lived sleptons, could travel with a speed measurably lower than the speed of light, giving a late arrival in the calorimeters and/or muon spectrometer, as well as high $\mathrm{dE} / \mathrm{dx}$ in the pixel detector. Hadron-like SMPs, such as R-hadrons, could exchange electric charge when penetrating the detector material and therefore possibly be dominantly neutral in the inner tracker or the muon spectrometer. Therefore, two strategies were prepared. The first one is designed to search for hadron-like SMPs using only the inner detector (ID) and calorimeter in ATLAS, without relying on the muon spectrometer (MS). The other is designed to search for lepton-like SMPs, such as long-lived sleptons, using both ID and MS. No excess is observed above the estimated SM background in the high mass region (Fig. 12) and lower limits, at $95 \% \mathrm{CL}$, are set on the mass of the long-lived particles. Long-lived staus in GMSB models are excluded up to a mass of $300 \mathrm{GeV}$ for $\tan \beta=5-20$ (Fig. 13). Directly produced long-lived sleptons are excluded up to a mass of $278 \mathrm{GeV}$. R-hadrons, composites of gluino (stop, sbottom) and light quarks, are excluded up to a mass of $985 \mathrm{GeV}$ (683 GeV, $612 \mathrm{GeV}$ ) (Fig. 14).

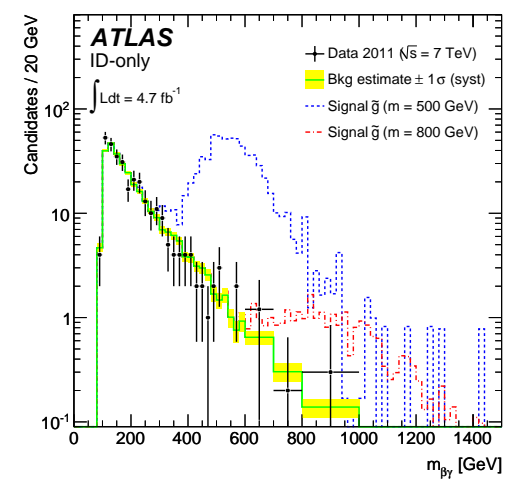

Figure 12. Observed data, background estimate and expected signal in the region for the lepton-like SMPs search [21].

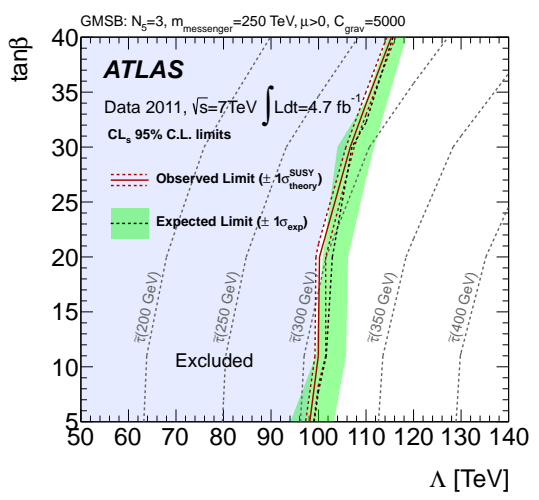

Figure 13. Limits on Lambda (SUSY-breaking mass scale) and tan beta in GMSB models. The observed and expected limit are shown, with the 1 sigma theoretical uncertainty and the 1 sigma band, respectively [21].

\section{Summary and Outlook}

We have presented the results of various searches for Rparity violating SUSY or long-lived particles at the ATLAS experiment. These searches need a full understanding of the detectors and "non-standard" analysis techniques. No excess beyond the Standard Model backgrounds have been seen yet, but all these analyses are being updated with full 2012 data and we continue to do our best to cover as much parameter space as we can. 


\section{References}

[1] H. Miyazawa, Prog. Theor. Phys. 36 (6), 1266 (1966)

[2] P. Ramond, Phys. Rev. D3, 2415 (1971)

[3] Y.A. Golfand, E.P. Likhtman, JETP Lett. 13, 323 (1971), [Pisma Zh. Eksp. Teor. Fiz. 13 (1971) 452455]

[4] A. Neveu, J.H. Schwarz, Nucl. Phys. B31, 86 (1971)

[5] A. Neveu, J.H. Schwarz, Phys. Rev. D4, 1109 (1971)

[6] J. Gervais, B. Sakita, Nucl. Phys. B34, 632 (1971)

[7] D.V. Volkov, V.P. Akulov, Phys. Lett. B46, 109 (1973)

[8] J. Wess, B. Zumino, Phys. Lett. B49, 52 (1974)

[9] J. Wess, B. Zumino, Nucl. Phys. B70, 39 (1974)

[10] M. Dine, W. Fischler, Phys. Lett. B110, 227 (1982)

[11] L. Alvarez-Gaume et al., Nucl. Phys. B207, 96 (1982)

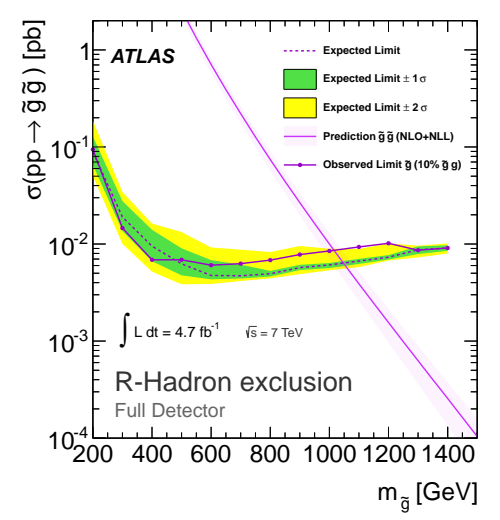

Figure 14. Cross-section upper limits at $95 \% \mathrm{CL}$ for gluino RHadrons [21]. Both the expected limit with \pm 1 sigma and \pm 2 sigma uncertainty bands and the observed upper limit are given. In addition the theoretical prediction for the production crosssection calculated at NLO+NLL and its uncertainty are drawn.
[12] ATLAS Collaboration, JINST 3, S08003 (2008)

[13] D. Alves et al., Nucl. Part. Phys. 39, 10 (2012)

[14] ATLAS Collaboration, ATLAS-CONF-2012-153 (2012), https://cds.cern.ch/record/1493492

[15] ATLAS Collaboration, hep-ex/1212.1272, submitted to PLB (2012)

[16] ATLAS Collaboration, JHEP 12, 86 (2012)

[17] ATLAS Collaboration, hep-ex/1210.2852, submmitted to PLB (2012)

[18] G.F. Giudice, M.A. Luty, H. Murayama, R. Rattazzi, JHEP 12, 027 (1998), hep-ph/9810442

[19] L. Randall, R. Sundrum, Nucl. Phys. B557, 79 (1999), hep-th/9810155

[20] ATLAS Collaboration, JHEP 01, 131 (2013)

[21] ATLAS Collaboration, hep-ex/1211.1597, submitted to PLB (2012) 